\title{
Lung cancer, immune thrombocytopenia and the lupus inhibitor
}

\author{
C.L. Kozlowski, ${ }^{1}$ M.J. Johnson, ${ }^{2}$ D.W. Gorst ${ }^{1}$ and R.F. Willey ${ }^{2}$ \\ Departments of ' Haematology and ${ }^{2}$ Medicine, Royal Lancaster Infirmary, Lancaster LA1 4RP, UK.
}

\begin{abstract}
Summary: This case report describes the simultaneous occurrence of autoimmune thrombocytopenia and the lupus anticoagulant in a patient with primary adenocarcinoma of the bronchus. Treatment with corticosteroids and plasmapheresis resulted in a return to normal of the partial thromboplastin time and the disappearance of the platelet antibodies. The carcinoma was inoperable.
\end{abstract}

\section{Introduction}

Both lupus anticoagulant and autoimmune thrombocytopenia may occur in association with malignancy. The lupus anticoagulant has been reported in patients with caecal, cervical, prostatic and metastatic adenocarcinomata. ${ }^{1,2}$ Autoimmune thrombocytopenia has been described in lymphoma and chronic lymphocytic leukaemia. ${ }^{3,4}$ We report both of these haematological disturbances in a patient with primary adenocarcinoma of the bronchus.

\section{Case report}

A 68 year old retired office worker presented with recent spontaneous epistaxis, bruising and a right hemiplegia. He also complained of left sided chest pain, cough and haemoptysis, which had failed to respond to three recent courses of antibiotics. He had lost a stone in weight and had smoked thirty cigarettes a day until 1967.

On examination, he was obese and had extensive bruising affecting his face, neck and right arm. There was a widespread purpuric rash with splinter haemorrhages of his finger nails. Air entry was reduced over the left upper zone of his chest and he had a right hemiplegia, mainly affecting his arm. Investigation showed a severe thrombocytopenia (platelets $8 \times 10^{9} / 1$ ) with a normal haemoglobin $(13.6 \mathrm{~g} / \mathrm{dl})$ and white cell count $7.8 \times 10^{9} / 1$ ). A coagulation screen was abnormal (partial thromboplastin time (PTT) 100 seconds, normal control 40 seconds) and an inhibitor test suggested a lupus anticoagulant. Anticardiolipin antibodies of both IgG and IgM isotypes were raised (IgG anticardiolipin units 40, IgM anticardiolipin units 40 . Normal range of each less than 10) and he had a false

Correspondence: C.L. Kozlowski, M.B., Ch.B.

Accepted: 24 March 1987 positive VDRL reaction. Fibrin degradation products were in the normal range and there was no evidence of fibrinolysis. Bone marrow aspiration demonstrated plentiful megakaryocytes suggesting immune thrombocytopenia and on presentation, platelet antibody was present. Antinuclear factor was negative and antiDNA antibodies were not found.

A chest X-ray showed dense opacification of the left upper zone and sputum cytology revealed clumps of adenocarcinoma cells. A computed tomographic scan of the brain indicated a probable haemorrhagic area in the left parietal region. An ultrasonic scan of the abdomen was normal. Oral prednisolone, $40 \mathrm{mg} /$ day was started and as there was no initial improvement in the platelet count or PTT, three 2 litre plasma exchanges were carried out. Bronchoscopy was then performed under platelet cover and an inoperable carcinoma involving the left lower trachea and the left main bronchus was found. Treatment with steroids was continued over a 9 month period, progressively reducing the dose.

The platelet count rose to $40 \times 10^{9} / 1$, the platelet antibody disappeared and the PTT returned to normal (see Figure 1). Movement in his right arm improved considerably and the patient had no further bruising or bleeding and felt well 9 months later.

\section{Methods}

Standard techniques ${ }^{5}$ were used for the haematological and coagulation tests. An inhibitor was suspected when mixing tests failed to correct a prolonged PTT. The test for lupus anticoagulant was carried out as described by Thomson. ${ }^{6}$ Platelet antibodies were detected using the ${ }^{51} \mathrm{Cr}$ labelled serotonin release test ${ }^{7}$ and ELISA method. ${ }^{8}$ The anticardiolipin antibodies were measured using a solid

(C) The Fellowship of Postgraduate Medicine, 1987 
phase radioimmunoassay technique. ${ }^{9}$ The VDRL test was a slide aggregation test using oxoid modified VDRL carbon antigen. The results of the platelet counts. test for platelet antibodies, PTT and tests for inhibitor are shown in Figure 1.

\section{Discussion}

The patient presented with autoimmune non-metastatic complications of his neoplasm. He was found to have the lupus anticoagulant, thrombocytopenia with platelet antibodies, IgG and IgM anticardiolipin antibodies and a false positive VDRL reaction in association with a bronchial adenocarcinoma.

The lupus anticoagulant is detected by its ability in vitro to cause abnormally prolonged coagulation tests (particularly the PTT) by inhibiting the prothrombin activation complex. ${ }^{10}$ Fifty percent of cases of lupus anticoagulant are associated with systemic lupus erythematosus and related autoimmune disorders. The other $50 \%$ have been found to occur in a wide range of diseases, including a few cases of malignant conditions; prostatic carcinoma, myeloma, Hodgkin's disease, cervical carcinoma, caecal carcinoma, lymphosarcoma ${ }^{1}$ and more recently hairy cell Leukaemia." The lupus anticoagulant is sometimes an incidental finding but paradoxically may also cause thrombotic episodes. ${ }^{12}$ Evidence suggests that it is an antiphospholipid antibody which reacts with negatively charged phospholipids on the platelet membrane causing damage, increased adhesion and subsequent thrombosis. Thrombocytopenia may also occur, possibly as a result of increased uptake and destruction by the reticuloendothelial system, following binding to the antiphospholipid antibody. ${ }^{13}$ Bleeding is not usually a feature of the lupus anticoagulant unless an additional factor is also present, coincidental thrombocytopenia, coagulation defect or vascular abnormality.

Since the presence of the lupus anticoagulant per se may lead to thrombocytopenia, the interpretation of the cause of clinical symptoms when both the lupus anticoagulant and autoimmune thrombocytopenia are present is complex.

Our patient's hemiplegia may have been due to a cerebral arterial thrombotic episode, associated with the lupus anticoagulant or to an intracerebral bleed, associated with his severe immune thrombocytopenia. Additional contributory factors, however, cannot be excluded. The fact that the hemiplegia recovered during steroid therapy, in association with the disappearance of both the lupus anticoagulant and platelet antibodies does not clarify the mechanism, but makes it seem, more likely that his stroke was due to thrombocytopenia and that a rise in platelet count above a critical level prevented further episodes of cerebrovascular symptoms.

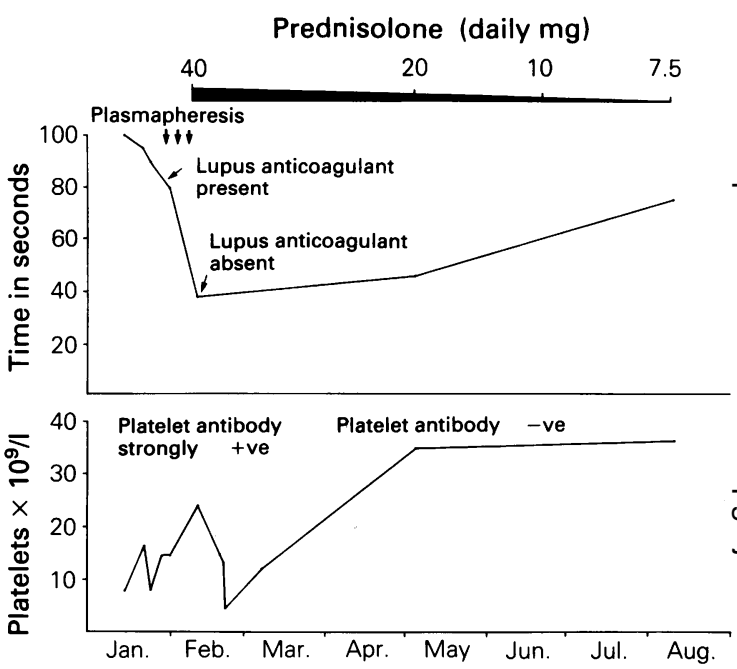

Figure 1 Improvement in the partial thromboplastin time (upper graph) and platelet count (lower graph) following treatment with oral prednisolone, with the disappearance of both the lupus anticoagulant and platelet antibody.

The patient's initial presentation with spontaneous bleeding is probably attributable to his severe throm bocytopenia and recovered when a stable, highes. platelet count was reached.

Anticardiolipin antibody is also thought to be antiphospholipid and a close correlation has been found between both IgG and IgM anticardiolipin antibodies and the lupus anticoagulant. ${ }^{13}$ Both are frequently accompanied by a false positive VDRL. Anticardiolipin antibody has also been described in the thrombocytopenia of systemic lupus erythematosus and related autoimmune disorders ${ }^{14}$ and in autoimmune thrombocytopenia although the correlation is less in the latter condition..$^{15}$

The four antibody reactions in our patient are most commonly found either individually or in combination, in patients suffering from systemic lupus erythematosus and other related autoimmune disorders. Antinuclear factor and anti-DNA antibodies in our patient remained persistently negative and no clinical evidence of systemic lupus erythematosus was found.

Autoimmune thrombocytopenia is commonly found in lymphoma and chronic lymphatic leukaemia but is uncommon in adenocarcinoma. We suggest that this patient's autoimmune manifestations are further examples of the paraneoplastic syndrome described by Grunwald et al.$^{16}$ and that the autoimmune response is stimulated by the tumour. This case study shows that although the patient's adenocarcinoma was inoperable, the distressing haematological symptoms disap- 
peared with treatment, reinforcing the value of low dose corticosteroids in the management of some cases of autoimmune complications associated with malignancy.

\section{References}

1. Schneider, M.A., Nachman, R.L., Jaffe, E.A. et al. A clinical study of the lupus anticoagulant. Blood 1976, 48: 499-508.

2. Mills, R.C., Zacharski, L.R. \& McIntyre, O.R. Circulating anticoagulant, auto-immune haemolytic anaemia and malignant lymphoma. Am J Med Sci 1977, 274: 1, 75.

3. Hoffbrand, A.V. \& Lewis, S.M. In Postgraduate Haematology, 5th edition. William Heinemann Medical Books, 1981, pp 484 and 650.

4. Ebbe, S., Wittels, B. \& Damashek, W. Auto-immune thrombocytopenic purpura ('ITP type') with chronic lymphatic leukaemia. Blood 1962, 19: 23-37.

5. Dacie, J.V. \& Lewis, S.M. In Practical Haematology, 6th edition, Churchill Livingstone, Edinburgh, 1984, pp 208-223.

6. Thomson, J.M. In Blood Coagulation and Haemostasis. A Practical Guide, 2nd edition, Churchill Livingstone, Edinburgh, 1981, pp 216.

7. Heinrich, D., Kessler, C. \& Mueller-Eckhardt, C. A comparative analysis of methods suitable for detection of platelet autoantibodies. Inhibition of $14 \mathrm{C}$ serotonin uptake, 14C serotonin release and microcomplement fixation. Vox Sang 1977, 32: 325-335.

8. Engvall, E. \& Perlman, P. Enzyme linked immunosorbent assay (ELISA). iii. Quantitation of specific antibodies by enzyme labelled immunosorbent antiimmunoglobulin in antigen coated tubes. $J$ Immunol 1972, 189: 129-135.

\section{Acknowledgements}

We thank Miss J. Fletcher for typing this report and the Immunology Departments in Lancaster and Preston for their assitance.

9. Harris, E.N., Gharavi, A.E., Boey, M.L. et al. Anticardiolipin antibodies, detection by radioimmunoassay and association with thrombosis in systemic lupus erythematosus. Lancet 1983, ii: 1211-1214.

10. Editorial. Lupus anticoagulant. Lancet 1984, $1157-$ 1158.

11. Duncombe, A.S., Dalton, R.G. \& Savidge, G.F. Lupus type coagulation inhibitor in hairy cell leukaemia and resolution with splenectomy. $\mathrm{Br} J$ Haematol 1987, 65: $120-121$.

12. Mueh, J.R., Herbst, K.D. \& Rappaport, S.I. Thrombosis in patients with the lupus anticoagulant. Ann Intern Med 1980, 92: 156-159.

13. Harris, E.N., Gharavi, A.E. \& Hughes, G.R.V. Antiphospholipid antibodies. Clin Rheum Dis 1985, 11, $591-$ 606.

14. Harris, E.N., Asherson, A.E., Gharavi, S.H. et al. Thrombocytopenia in S.L.E. and related autoimmune disorders: association with anticardiolipin antibody. $\mathrm{Br} J$ Haematol 1985, 59: 227-230.

15. Harris, E.N., Gharavi, A.E., Heyde, U. et al. Anticardiolipin antibodies in autoimmune thrombocytopenic purpura. Br J Haematol 1985, 59: 231-234.

16. Grunwald, G.B., Klein, R., Simmonds, M.A. et al. Autoimmune basis for paraneoplastic syndrome in patients with small cell lung carcinoma. Lancet 1985, i: $658-661$. 